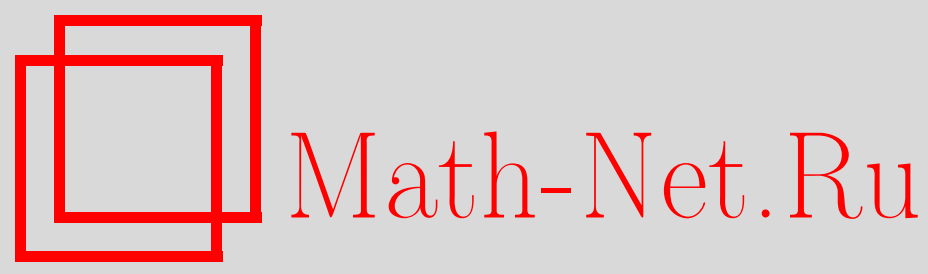

Д. Ф. Гогуадзе, О понятии полукольца множеств, Матем. заметки, 2003, том 74, выпуск 3, 362-368

DOI: https://doi.org/10.4213/mzm270

Использование Общероссийского математического портала Math-Net.Ru подразумевает, что вы прочитали и согласны с пользовательским соглашением http://www.mathnet.ru/rus/agreement

Параметры загрузки:

IP : 3.95.254.165

26 апреля 2023 г., 16:26:50

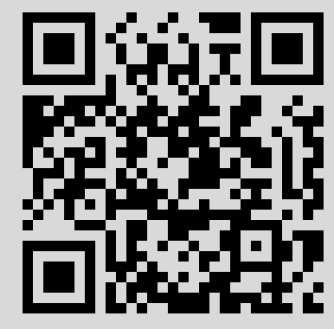




\title{
О ПОНЯТИИ ПОЛУКОЛЬЦА МНОЖЕСТВ
}

\author{
Д. Ф. Гогуадзе
}

В заметке вводится обобщенное понятие полукольца и доказьвается, что оно обладает всеми известными свойствами полукольца согласно старому определению.

Библиограффия: 2 названия.

Понятие полукольца является исходной точкой для современной теории меры и интеграла. Но в определении полукольца пересечение и разность множеств выступают не в равноправном виде.

В настоящей заметке вводится обобщенное понятие полукольца, в котором пересечение и разность множеств представлены в одинаковой форме и доказывается, что оно в теории меры исполняет ту же самую роль, что и старое понятие.

В дальнейшем мы в основном пользуемся обозначениями и терминами из [1] и [2].

Пусть $\mathfrak{A}$ - произвольный класс множеств и $E \in \mathfrak{A}$. Конечньй класс попарно непересекающихся множеств $\left\{E_{1}, \ldots, E_{n}\right\} \subset \mathfrak{A}$, объединение которых равно $E$, назьвается разбиением множества $E$ и обозначается через $D E$. При этом, множества $E_{k}, k=1, \ldots, n$, назьваются компонентами разбиения $D E$. Разбиение $D_{1} E$ называется продолжением разбиения $D E$, если каждьй компонент разбиения $D_{1} E$ является подмножеством некоторого компонента разбиения $D E$ или, что то же самое, если разбиение $D_{1} E$ содержит разбиение каждого компонента разбиения $D E$.

Класс множеств, содержаший вместе с любьми двумя своими множествами $E$ и $F$ и их пересечение $E \cap F$, называется мультипликативным и обозначается через $\mathfrak{M}_{0}$.

Класс множеств, содержащий вместе с любыми двумя своими множествами $E$ и $F$ и разбиение их пересечения $E \cap F$, назовем обобщенно мультипликативным и обозначим через $\mathfrak{M}$. Если $D_{1} E=\left\{E_{1}^{\prime}, \ldots, E_{m}^{\prime}\right\}$ и $D_{2} E=\left\{E_{1}^{\prime \prime}, \ldots, E_{n}^{\prime \prime}\right\}$ - два разбиения множества $E \in \mathfrak{M}$, то $\left\{E_{k}^{i j}\right\} \quad\left(k=1, \ldots, N_{i j} ; i=1, \ldots, m ; j=1, \ldots, n\right)$, где $\left\{E_{k}^{i j}\right\}$, $k=1, \ldots, N_{i j}$, является разбиением пересечения $E_{i}^{\prime} \cap E_{j}^{\prime \prime}$, назьвается произведением разбиений $D_{1} E$ и $D_{2} E$ и обозначается через $\left(D_{1} \cdot D_{2}\right) E$. Оно, очевидно, является продолжением обоих разбиений.

Обозначим через $\mathfrak{M} E$ множество всех компонентов всевозможных разбиений множества $E \in \mathfrak{M}$.

Приведем теперь старое, а затем новое определение полукольца.

Непустой класс $P_{0}$ назьвается полукольцом, если он обладает следующими двумя свойствами:

1) если $A \in P$ и $B \in P_{0}$, то $A \cap B \in P_{0}$;

2) если $A, B \in P_{0}$ и $A \supset B$, то $P_{0}$ содержит разбиение разности $A-B$. 
Непустой класс $P$ будем называть полукольцом, если он обладает следующими двумя свойствами:

1) если $A \in P$ и $B \in P$, то он содержит разбиение их пересечения $A \cap B$;

2) если $A, B \in P$ и $A \supset B$, то он содержит разбиение разности $A-B$.

Таким образом, в новом определении полукольца мультипликативность класса заменена его обобщенной мультипликативностью.

Рассмотрим класс

$$
P=\{\{1,2,3,4\},\{1,2,3\},\{2,3,4\},\{1\},\{2\},\{3\},\{4\}, \varnothing\},
$$

где $\varnothing$ обозначает пустое множество. Тогда

$$
\{1,2,3\} \cap\{2,3,4\}=\{2,3\} \bar{\in} P,
$$

однако

$$
\{1,2,3\} \cap\{2,3,4\}=\{2\} \cup\{3\},
$$

а это разбиение $\{\{2\},\{3\}\}$ множества $\{2,3\}$ содержится в $P$.

Поэтому легко проверить непосредственно, что класс $P$ не является полукольцом по старому определению, но является им согласно новому определению.

Пусть $E$ - счетное множество и $T(E)$ - класс всех его подмножеств. Возьмем конечное подмножество $e=\left\{x_{1}, \ldots, x_{n}\right\}$ множества $E$ и из класса $T(E)$ удалим множество $e$ и все его подмножества, кроме всех одноэлементных подмножеств. Обозначим через $P$ оставшийся класс. Покажем, что он является обобщенно мультипликативньм, но не мультипликативным. Действительно, возьмем множество $E-e$ и представим его в виде $E-e=F_{1} \cup F_{2}$, где $F_{1} \cap F_{2}=\varnothing$. Образуем множества $E_{1}=F_{1} \cup e$ и $E_{2}=F_{2} \cup e$. Тогда $E_{1}, E_{2} \in P$ и $E_{1} \cap E_{2}=\left(F_{1} \cup e\right) \cap\left(F_{2} \cup e\right)=e$. Следовательно, $E_{1} \cap E_{2} \bar{\epsilon} P$. Однако $P$ содержит конечное разбиение $\left\{\left\{x_{1}\right\}, \ldots,\left\{x_{n}\right\}\right\}$ множества $E_{1} \cap E_{2}=e$.

То, что $P$ удовлетворяет второму условию полукольца, тривиально проверяется, так как, если при взятии разности двух множеств из $P$ получаются множество $e$ или его подмножества, то их разбиения содержатся в $P$.

Таким образом, $P$ является полукольцом согласно новому определению, но не согласно старому определению.

Докажем основные свойства полукольца.

Всякое полукольцо $P$ содержит пустое множество. Это непосредственно следует из непустоты класса $P$ и условия 2.

ПРЕДЛОЖЕНИЕ 1. Если $A_{1}, \ldots, A_{n}$ - попарно непересекающиеся множества из полукольиа $P$, содержащиеся в некотором множестве $A \in P$, то найдутся такие множества $B_{1}, \ldots, B_{m} \in P$, что

$$
A=A_{1} \cup \cdots \cup A_{n} \cup B_{1} \cup \cdots \cup B_{m},
$$

причем все мнохества справа также попарно не пересекаются, т.е. класс $\left\{A_{1}, \ldots, A_{n}, B_{1}, \ldots, B_{m}\right\}$ является разбиением множества $A$. 
ДокАЗАТЕЛЬство. Для $n=1$ предложение непосредственно следует из свойства 2 полукольца $P$. Допустим, что оно справедливо для некоторого $n$ и докажем его для $n+1$. Согласно допущению существует представление (1). Так как множество $A_{n+1}$ содержится в $A$ и не пересекается с $A_{1}, \ldots, A_{n}$, в силу первого свойства полукольца $P$ имеем

$$
A_{n+1}=\bigcup_{k=1}^{m}\left(A_{n+1} \cap B_{k}\right)=\bigcup_{k=1}^{m} \bigcup_{i=1}^{q_{k}} C_{i}^{k} .
$$

$\mathrm{Ho}$

$$
B_{k}=\left[B_{k}-\left(A_{n+1} \cap B_{k}\right)\right] \cup\left(A_{n+1} \cap B_{k}\right),
$$

и согласно второму свойству полукольца $P$ имеем

$$
B_{k}-\left(A_{n+1} \cap B_{k}\right)=\bigcup_{i=1}^{r_{k}} D_{i}^{k} .
$$

Поэтому

$$
\begin{aligned}
\bigcup_{k=1}^{m} B_{k} & =\bigcup_{k=1}^{m}\left(\left[B_{k}-\left(A_{n+1} \cap B_{k}\right)\right] \cup\left(A_{n+1} \cap B_{k}\right)\right) \\
& =\bigcup_{k=1}^{m}\left[B_{k}-\left(A_{n+1} \cap B_{k}\right)\right] \cup \bigcup_{k=1}^{m}\left(A_{n+1} \cap B_{k}\right) \\
& =\bigcup_{k=1}^{m} \bigcup_{i=1}^{r_{k}} D_{i}^{k} \cup \bigcup_{k=1}^{m} \bigcup_{i=1}^{q_{k}} C_{i}^{k}=\bigcup_{k=1}^{m} \bigcup_{i=1}^{r_{k}} D_{i}^{k} \cup A_{n+1} .
\end{aligned}
$$

Таким образом, подставляя это равенство в формулу (1), получим

$$
A=A_{1} \cup \cdots \cup A_{n} \cup A_{n+1} \cup D_{1}^{1} \cup \cdots \cup D_{r_{1}}^{1} \cup \cdots \cup D_{1}^{m} \cup \cdots \cup D_{r_{m}}^{m},
$$

причем все множества справа принадлежат полукольцу $P$ и попарно не пересекаются.

ПРЕДЛОЖЕНИЕ 2. Если $A_{1}, \ldots, A_{m}$ - произвольный конечный класс множсеств из полукольца $P$, то их обвединение можно представить в виде

$$
A_{1} \cup \cdots \cup A_{m}=A_{1}^{1} \cup \cdots \cup A_{1}^{k_{1}} \cup \cdots \cup A_{m}^{1} \cup \cdots \cup A_{m}^{k_{m}},
$$

әде $A_{i}^{1}, \ldots, A_{i}^{k_{i}}$ содержатся в множестве $A_{i}, \quad i=1, \ldots, m, и$ все множества справа принадлежат полукольиу $P$ и попарно не пересекаются.

ДокАЗАТЕЛЬСтво. Для $m=1$ предложение очевидно. Допустим, что оно верно для $m$ и докажем его для $m+1$. По предположению имеет место равенство $(2)$. В силу первого свойства полукольца $P$ существуют представления

$$
\begin{aligned}
A_{m+1} \cap A_{1}^{1} & =\bigcup_{i=1}^{n_{1}} B_{i}^{1}, \quad \ldots, \quad A_{m+1} \cap A_{1}^{k_{1}}=\bigcup_{i=1}^{n_{k_{1}}} B_{i}^{k_{1}}, \quad \ldots, \\
A_{m+1} \cap A_{m}^{1} & =\bigcup_{i=1}^{q_{1}} C_{i}^{1}, \quad \ldots, \quad A_{m+1} \cap A_{m}^{k_{m}}=\bigcup_{i=1}^{q_{k_{m}}} C_{i}^{k_{m}} .
\end{aligned}
$$


Согласно предложению 1 сушествует представление

$$
A_{m+1}=\bigcup_{i=1}^{n_{1}} B_{i}^{1} \cup \cdots \cup \bigcup_{i=1}^{n_{k_{1}}} B_{i}^{k_{1}} \cup \cdots \cup \bigcup_{i=1}^{q_{k}} C_{i}^{1} \cup \cdots \cup \bigcup_{i=1}^{q_{k_{m}}} C_{i}^{k_{m}} \cup A_{m+1}^{1} \cup \cdots \cup A_{m+1}^{k_{m+1}}
$$

причем слагаемые справа принадлежат полукольцу $P$ и попарно не пересекаются. Учитывая эти соотношения, из (2) получаем

$$
\begin{aligned}
A_{1} \cup \cdots \cup A_{m} \cup A_{m+1}= & A_{1}^{1} \cup \cdots \cup A_{1}^{k_{1}} \cup \cdots \cup A_{m}^{1} \cup \cdots \cup A_{m}^{k_{m}} \\
& \cup \bigcup_{i=1}^{n_{1}} B_{i}^{1} \cup \cdots \cup \bigcup_{i=1}^{n_{k_{1}}} B_{i}^{k_{1}} \cup \cdots \cup \bigcup_{i=1}^{q_{1}} C_{i}^{1} \cup \cdots \cup \bigcup_{i=1}^{q_{k_{m}}} C_{i}^{k_{m}} \\
& \cup A_{m+1}^{1} \cup \cdots \cup A_{m+1}^{k_{m+1}} \\
= & A_{1}^{1} \cup \cdots \cup A_{1}^{k_{1}} \cup \cdots \cup A_{m}^{1} \cup \cdots \cup A_{m}^{k_{m}} \cup A_{m+1}^{1} \cup \cdots \cup A_{m+1}^{k_{m+1}},
\end{aligned}
$$

причем слагаемые справа вновь принадлежат полукольцу $P$ и вновь попарно не пересекаются.

Теорема 1. Если $P$ - полукольцо, то наименьшее кольио $R(P)$, порохсденное полукольиом $P$, совпадает с классом $K$ всех мнохеств $A$, имеющих вид

$$
A=\bigcup_{k=1}^{n} A_{k}
$$

где все множества $A_{k}(k=1, \ldots, n)$ принадлежат полукольиу $P$ и попарно не пересекаются.

ДокАЗАТЕЛЬСТво. Покажем, что класс $K$ образует кольцо. Для этого достаточно показать, что класс $K$ замкнут относительно образования пересечений и симметрических разностей двух множеств (см. [1, с. 38] или [2, с. 26]).

Если $A$ и $B$ принадлежат классу $K$, то

$$
A=\bigcup_{i=1}^{n} A_{i} \quad \text { и } \quad B=\bigcup_{j=1}^{m} B_{j}
$$

где $A_{i}, i=1, \ldots, n$, соответственно $B_{j}, j=1, \ldots, m$, являются попарно непересекающимися множествами из полукольца $P$.

В силу первого свойства полукольца $P$ имеем

$$
A_{i} \cap B_{j}=\bigcup_{k=1}^{n_{i j}} C_{k}^{i j}
$$

где $C_{k}^{i j}, k=1, \ldots, n_{i j}$, представляют собой попарно непересекаюшиеся множества из полукольца $P$. 
Согласно предложению 1 имеют место равенства

$$
A_{i}=\bigcup_{j=1}^{m} \bigcup_{k=1}^{n_{i j}} C_{k}^{i j} \cup \bigcup_{k=1}^{r_{i}} D_{i k}, \quad B_{j}=\bigcup_{i=1}^{n} \bigcup_{k=1}^{n_{i j}} C_{k}^{i j} \cup \bigcup_{t=1}^{S_{j}} E_{i t},
$$

где $D_{i k}, k=1, \ldots, r_{i}$, соответственно $E_{j t}, t=1, \ldots, s_{j}$, являются попарно непересекающимися множествами из полукольца $P$. Из этих равенств получаем

$$
\begin{gathered}
A=\bigcup_{i=1}^{n} A_{i}=\bigcup_{i=1}^{n} \bigcup_{j=1}^{m} \bigcup_{k=1}^{n_{i j}} C_{k}^{i j} \cup \bigcup_{i=1}^{n} \bigcup_{k=1}^{r_{i}} D_{i k}, \\
B=\bigcup_{j=1}^{m} \bigcup_{i=1}^{n} \bigcup_{k=1}^{n_{i j}} C_{k}^{i j} \cup \bigcup_{j=1}^{m} \bigcup_{t=1}^{S_{j}} E_{i t} .
\end{gathered}
$$

Отсюда, в свою очередь, следует

$$
A \cap B=\bigcup_{i=1}^{n} \bigcup_{j=1}^{m} \bigcup_{k=1}^{n_{i j}} C_{k}^{i j}, \quad A \triangle B=\bigcup_{i=1}^{n} \bigcup_{k=1}^{r_{i}} D_{i k} \cup \bigcup_{i=1}^{m} \bigcup_{t=1}^{s_{j}} E_{j t},
$$

что означает $A \cap B \in K$ и $A \triangle B \in K$. Следовательно, класс $K$ действительно представляет собой кольцо. То, что оно является наименьшим, очевидно.

TеОрема 2. Пусть $\mathfrak{M}$ - обобщенно мультипликативный класс и $E_{0} \in \mathfrak{M}$. Тогда класс $\mathfrak{M} E_{0}$ всех компонентов всевозможсны разбиений множества $E_{0}$ представляет собой полукольцо.

ДокАЗАТЕЛЬСтво. Пусть $E, F \in \mathfrak{M} E_{0}$. Тогда найдутся такие разбиения $D_{1} E_{0}$ и $D_{2} E_{0}$, что $E \in D_{1} E_{0}$ и $F \in D_{2} E_{0}$. Кроме того, так как класс $\mathfrak{M}$ является обобщенно мультипликативным,

$$
E \cap F=\bigcup_{k=1}^{n} e_{k}
$$

Образуем произведение $\left(D_{1} \cdot D_{2}\right) E_{0}$ разбиений $D_{1} E_{0}$ и $D_{2} E_{0}$. Тогда согласно определению произведения разбиений $\left\{e_{1}, e_{2}, \ldots, e_{n}\right\} \subset\left(D_{1} \cdot D_{2}\right) E_{0}$. Следовательно, $\left\{e_{1}, e_{2}, \ldots\right.$, $\left.e_{n}\right\} \subset \mathfrak{M} E_{0}$.

Таким образом, класс $\mathfrak{M} E_{0}$ удовлетворяет первому условию определения полукольца.

Пусть теперь $E, F \in \mathfrak{M} E_{0}$ и $E \subset F$. Тогда найдутся такие разбиения $D_{1} E_{0}$ и $D_{2} E_{0}$, что $E \in D_{1} E_{0}$ и $F \in D_{2} E_{0}$. Образуем их произведение $\left(D_{1} \cdot D_{2}\right) E_{0}$. Тогда $E \cap F=E \in$ $\left(D_{1} \cdot D_{2}\right) E_{0}$, и так как $\left(D_{1} \cdot D_{2}\right) E_{0}$ является продолжением разбиения $D_{2} E_{0}$, то $\left(D_{1}\right.$. $\left.D_{2}\right) E_{0}$ содержит разбиение множества $F$, компонентом которого является множество $E$. Следовательно, класс $\mathfrak{M} E_{0}$ удовлетворяет и второму условию определения полукольца.

Пусть $X, Y$-произвольные множества. Совокупность всех упорядоченных пар $(x, y)$, где $x \in X$ и $y \in Y$, назьвается декартовым произведением множеств $X$ и $Y$ и обозначается через $X \times Y$. Если $A \subset X$ и $B \subset Y$, то множество $E=A \times B$, содержащееся в $X \times Y$, назьвается прямоугольником, а сами множества $A$ и $B-$ сторонами этого прямоугольника.

Если $\mathfrak{A}$ и $\mathfrak{B}$ - классы множеств, то совокупность всех прямоугольников $A \times B$, где $A \in \mathfrak{A}$ и $B \in \mathfrak{B}$, назьвается произведением классов $\mathfrak{A}$ и $\mathfrak{B}$ и обозначается через $\mathfrak{A} \otimes \mathfrak{B}$.

Нам в дальнейшем понадобится следующая простая 
Лемма. Непустые прямоугольники $A_{1} \times B_{1} u A_{2} \times B_{2}$ являются непересекающимися тогда и только тогда, когда или $A_{1} \cap A_{2}=\varnothing$ или $B_{1} \cap B_{2}=\varnothing$.

ДокАЗАТЕЛЬСтво. Необходимость. Пусть

$$
\left(A_{1} \times B_{1}\right) \cap\left(A_{2} \times B_{2}\right)=\varnothing,
$$

но и $A_{1} \cap A_{2} \neq \varnothing$ и $B_{1} \cap B_{2} \neq \varnothing$. Пусть $x \in A_{1} \cap A_{2}$ и $y \in B_{1} \cap B_{2}$. Тогда

$$
(x, y) \in\left(A_{1} \cap A_{2}\right) \times\left(B_{1} \cap B_{2}\right)=\left(A_{1} \times B_{1}\right) \cap\left(A_{2} \times B_{2}\right)=\varnothing,
$$

что невозможно.

Достаточность. Пусть или $A_{1} \cap A_{2}=\varnothing$ или $B_{1} \cap B_{2}=\varnothing$, но

$$
\left(A_{1} \times B_{1}\right) \cap\left(A_{2} \times B_{2}\right) \neq \varnothing
$$

и

$$
(x, y) \in\left(A_{1} \times B_{1}\right) \cap\left(A_{2} \times B_{2}\right)=\left(A_{1} \cap A_{2}\right) \times\left(B_{1} \cap B_{2}\right) .
$$

Отсюда $x \in A_{1} \cap A_{2}$ и $y \in B_{1} \cap B_{2}$, что невозможно.

Теорема 3. Если $P_{1}$ и $P_{2}-$ полукольиа, то их произведение $P=P_{1} \otimes P_{2}$ также является полукольиом.

ДокАЗАТЕЛЬСТво. Пусть $E_{1}, E_{2} \in P$. Тогда

$$
E_{1}=A_{1} \times B_{1}, \quad E_{2}=A_{2} \times B_{2} \quad\left(A_{1}, A_{2} \in P_{1}, \quad B_{1}, B_{2} \in P_{2}\right) .
$$

Кроме того

$$
E_{1} \cap E_{2}=\left(A_{1} \times B_{1}\right) \cap\left(A_{2} \times B_{2}\right)=\left(A_{1} \cap A_{2}\right) \times\left(B_{1} \cap B_{2}\right) .
$$

Так как $P_{1}$ и $P_{2}$ являются полукольцами, то

$$
A_{1} \cap A_{2}=\bigcup_{i=1}^{m} C_{i}, \quad B_{1} \cap B_{2}=\bigcup_{k=1}^{n} D_{k},
$$

где $C_{i}, i=1, \ldots, m,-$ попарно непересекающиеся множества из полукольца $P_{1}$ и $D_{k}$, $k=1, \ldots, n,-$ аналогичные множества из полукольца $P_{2}$. Поэтому имеем

$$
E_{1} \cap E_{2}=\bigcup_{i=1}^{m} C_{i} \times \bigcup_{k=1}^{n} D_{k}=\bigcup_{i=1}^{m} \bigcup_{k=1}^{n}\left(C_{i} \times D_{k}\right),
$$

где согласно лемме $C_{i} \times D_{k}, i=1, \ldots, m, k=1, \ldots, n$, попарно непересекающиеся прямоугольники из $P$.

Следовательно, класс $P$ удовлетворяет первому условию определения полукольца.

Пусть теперь $E_{1}, E_{2} \in P\left(E_{1}=A_{1} \times B_{1}, E_{2}=A_{2} \times B_{2}\right)$ - непустые прямоугольники и $E_{1} \subset E_{2}$. Тогда (см. [2, с. 137]) $A_{1} \subset A_{2}$ и $B_{1} \subset B_{2}$. В силу того, что $P_{1}$ и $P_{2}$ являются полукольцами, имеют место представления

$$
A_{2}=A_{1} \cup A^{1} \cup \cdots \cup A^{m}, \quad B_{2}=B_{1} \cup B^{1} \cup \cdots \cup B^{n} .
$$


Отсюда получаем

$$
\begin{aligned}
A_{2} \times B_{2}= & \left(A_{1} \cup A^{1} \cup \cdots \cup A^{m}\right) \times\left(B_{1} \cup B^{1} \cup \cdots \cup B^{n}\right) \\
= & \left(A_{1} \times B_{1}\right) \cup\left(A_{1} \times B^{1}\right) \cup \cdots \cup\left(A_{1} \times B^{n}\right) \\
& \cup\left(A^{1} \times B_{1}\right) \cup\left(A^{1} \times B^{1}\right) \cup \cdots \cup\left(A^{1} \times B^{n}\right) \\
& \cdots \cdots \cdots \\
& \cup\left(A^{m} \times B_{1}\right) \cup\left(A^{m} \times B^{1}\right) \cup \cdots \cup\left(A^{m} \times B^{n}\right),
\end{aligned}
$$

причем все прямоугольники справа принадлежат $P$ и согласно лемме попарно не пересекаются.

Следовательно, класс $P$ удовлетворяет и второму условию определения полукольца.

Таким образом, полукольцо, согласно новому определению, обладает всеми известными свойствами полукольца по старому определению.

\section{СПИСОК ЦИТИРОВАННОЙ ЛИТЕРАТУРЫ}

[1] Колмогоров А. Н., Фомин С. В. Элементы теории функций и функционального анализа. M.: Наука, 1972.

[2] Халмош П. Теория меры. М.: ИЛ, 1953.

Институт вычислительной математики им. Н. Мусхелишвили, 